\title{
A Numerical Investigation on the Structure of the Zeros of Euler Polynomials
}

\author{
C. S. Ryoo and J. Y. Kang \\ Department of Mathematics, Hannam University, Daejeon 306-791, Republic of Korea \\ Correspondence should be addressed to J. Y. Kang; rkdwjddnr2002@yahoo.co.kr \\ Received 13 January 2015; Accepted 17 May 2015 \\ Academic Editor: Guang Zhang
}

Copyright ( 2015 C. S. Ryoo and J. Y. Kang. This is an open access article distributed under the Creative Commons Attribution License, which permits unrestricted use, distribution, and reproduction in any medium, provided the original work is properly cited.

Using numerical investigation, we observe the behavior of complex roots of the Euler polynomials $E_{n}(x)$. By means of numerical experiments, we demonstrate a remarkably regular structure of the complex roots of the Euler polynomials $E_{n}(x)$. Finally, we show the Julia set of Newton iteration function $R(x)=x-E_{n}(x) / E_{n}^{\prime}(x)$.

\section{Introduction}

The computing environment would make more and more rapid progress and there has been increasing interest in solving mathematical problems with the aid of computers. By using software, mathematicians can explore concepts much more easily than in the past. The ability to create and manipulate figures on the computer screen enables mathematicians to quickly visualize and produce many problems, examine properties of the figures, look for patterns, and make conjectures. This capability is especially exciting because these steps are essential for most mathematicians to truly understand even basic concept. Numerical experiments of Bernoulli polynomials, Euler polynomials, and Genocchi polynomials have been the subject of extensive study in recent year and much progress has been made both mathematically and computationally. Mathematicians have studied different kinds of the Euler, Bernoulli, Tangent, and Genocchi numbers and polynomials. Recently, many authors have studied in the area of the $q$-analogues of these numbers and polynomials (see [1-20]). Using computer, a realistic study for the zeros of Euler polynomials $E_{n}(x)$ is very interesting. The main purpose of this paper is to observe an interesting phenomenon of "scattering" of the zeros of the Euler polynomials $E_{n}(x)$ in complex plane. Throughout this paper, we always make use of the following notations: $\mathbb{N}=\{1,2,3, \ldots\}$ denotes the set of natural numbers, $\mathbb{N}_{0}=\{0,1,2,3, \ldots\}$ denotes the set of nonnegative integers, $\mathbb{Z}$ denotes the set of integers, $\mathbb{R}$ denotes the set of real numbers, and $\mathbb{C}$ denotes the set of complex numbers.

The classical Euler numbers $E_{n}$ and Euler polynomials $E_{n}(x)$ are usually defined by the following generating functions:

$$
\begin{aligned}
\left(\frac{2}{e^{t}+1}\right) & =\sum_{n=0}^{\infty} E_{n} \frac{t^{n}}{n !}, \\
\left(\frac{2}{e^{t}+1}\right) e^{x t} & =\sum_{n=0}^{\infty} E_{n}(x) \frac{t^{n}}{n !},
\end{aligned}
$$

where we use the technique method notation by replacing $E(x)^{n}$ by $E_{n}(x)$ symbolically.

Clearly, $E_{n}=E_{n}(0)$. These polynomials and numbers play important roles in many different areas of mathematics such as combinatorics, number theory, special function, and analysis, and numerous interesting results for them have been explored (see $[2,3,7-9,13,15,18-20])$. The following elementary properties of Euler polynomials $E_{n}(x)$ are readily derived from (1) and (2). We, therefore, choose to omit the details involved. For more studies and results in this subject we may see $[2,3,8,13,15,17-20]$. 
Theorem 1. For $n \in \mathbb{N}_{0}$, one has

$$
E_{n}(x)=\sum_{l=0}^{n}\left(\begin{array}{l}
n \\
l
\end{array}\right) E_{l} x^{n-l}
$$

By Theorem 1, after some elementary calculations, we get

$$
\begin{aligned}
\int_{a}^{b} E(x) d x & =\sum_{l=0}^{n}\left(\begin{array}{l}
n \\
l
\end{array}\right) E_{l} \int_{a}^{b} x^{n-l} d x \\
& =\left.\sum_{l=0}^{n}\left(\begin{array}{l}
n \\
l
\end{array}\right) E_{l} \frac{x^{n-l+1}}{n-l+1}\right|_{a} ^{b} \\
& =\left.\frac{1}{n+1} \sum_{l=0}^{n+1}\left(\begin{array}{c}
n+1 \\
l
\end{array}\right) E_{l} x^{n-l+1}\right|_{a} ^{b}
\end{aligned}
$$

By Theorem 1, we have

$$
\int_{a}^{b} E_{n}(x) d x=\frac{E_{n+1}(b)-E_{n+1}(a)}{n+1} .
$$

Since $E_{n}(0)=E_{n}$, by (5), we have the following theorem.

Theorem 2. For $n \in \mathbb{N}$, one has

$$
E_{n}(x)=E_{n}+n \int_{0}^{x} E_{n-1}(t) d t
$$

Then, it is easy to deduce that $E_{k}(x)$ are polynomials of degree $k$. Here is the list of the first Euler polynomials:

$$
\begin{aligned}
& E_{0}(x)=1 \\
& E_{1}(x)=x-\frac{1}{2} \\
& E_{2}(x)=x^{2}-x \\
& E_{3}(x)=x^{3}-\frac{3 x^{2}}{2}+\frac{1}{4} \\
& E_{4}(x)=x^{4}-2 x^{3}+x \\
& E_{5}(x)=x^{5}-\frac{5 x^{4}}{2}+\frac{5 x^{2}}{2}-\frac{1}{2} \\
& E_{6}(x)=x^{6}-3 x^{5}+5 x^{3}-3 x \\
& E_{7}(x)=x^{7}-\frac{7 x^{6}}{2}+\frac{35 x^{4}}{4}-\frac{21 x^{2}}{2}+\frac{17}{8} \\
& E_{8}(x)=x^{8}-4 x^{7}+14 x^{5}-28 x^{3}+17 x, \\
& E_{9}(x)=x^{9}-\frac{9 x^{8}}{2}+21 x^{6}-63 x^{4}+\frac{153 x^{2}}{2}-\frac{31}{2}, \\
& E_{10}(x)=x^{10}-5 x^{9}+30 x^{7}-126 x^{5}+255 x^{3}-155 x
\end{aligned}
$$

\section{The Phenomenon of Scattering of Zeros}

In this section, an interesting phenomenon of scattering of zeros of $E_{n}(x)$ is observed. By (2), we obtain

$$
\begin{aligned}
\sum_{n=0}^{\infty} E_{n}(1-x) \frac{(-1)^{n} t^{n}}{n !} & =\frac{2}{e^{-t}+1} e^{(1-x)(-t)}=\frac{2}{e^{t}+1} e^{x t} \\
& =\sum_{n=0}^{\infty} E_{n}(x) \frac{t^{n}}{n !} .
\end{aligned}
$$

Hence we have the following theorem.

Theorem 3 (see $[13,15]$ ). For $n \in \mathbb{N}_{0}$, one has

$$
E_{n}(x)=(-1)^{n} E_{n}(1-x) .
$$

By (9), we also have the following theorem.

Theorem 4. For $n \in \mathbb{N}_{0}$, if $n \equiv 1(\bmod 2)$, then $E_{n}(1 / 2)=0$; if $n \equiv 0(\bmod 2)$, then $E_{n}(0)=E_{n}(1)=0$.

In $[13,15]$, we made a series of the following conjectures.

Conjecture 5. Prove that $E_{n}(x), x \in \mathbb{C}$, has $\operatorname{Re}(x)=1 / 2$ reflection symmetry in addition to the usual $\operatorname{Im}(x)=0$ reflection symmetry analytic complex functions.

Conjecture 6. Prove that $E_{n}(x)=0$ has $n$ distinct solutions.

We find a counterexample of Conjecture 6. Let $n=5$. Then there are five numbers, $x_{i}(i=1,2,3,4,5)$, such that $E_{5}\left(x_{i}\right)=0$. That is, we obtain $x_{1}=1 / 2, x_{2}=(1 / 2)(1-$ $\sqrt{5}), x_{3}=(1 / 2)(1-\sqrt{5}), x_{4}=(1 / 2)(1+\sqrt{5})$, and $x_{5}=$ $(1 / 2)(1+\sqrt{5})$. Hence, Conjecture 6 is not true for all $n$. Using computers, many more values of $n$ have been checked. It still remains unknown if the conjecture fails or holds for any value $n \neq 5$.

Since $n$ is the degree of the polynomial $E_{n}(x)$, the number of real zeros $R_{E_{n}(x)}$ lying on the real plane $\operatorname{Im}(x)=0$ is then $R_{E_{n}(x)}=n-C_{E_{n}(x)}$, where $C_{E_{n}(x)}$ denotes complex zeros. See Table 1 for tabulated values of $R_{E_{n}(x)}$ and $C_{E_{n}(x)}$.

Conjecture 7. Prove that the number of complex zeros $C_{E_{n}(x)}$ of $E_{n}(x), \operatorname{Im}(x) \neq 0$ is

$$
\begin{aligned}
C_{E_{n}(x)} & =4\left[\frac{n-\alpha}{5}\right], \\
\alpha & =\left[\frac{n+20}{21}\right],
\end{aligned}
$$

where [ ] denotes taking the integer part.

Subsequently, much theoretical and computational work has been done, extending and testing these conjectures, with particular attention paid recently to certain refined conjectures for the analytic continued Euler polynomials (see [15]). By means of numerical experiments, we observe that $E_{n}(x), x \in \mathbb{C}$, has $\operatorname{Re}(x)=1 / 2$ reflection symmetry in addition to the usual $\operatorname{Im}(x)=0$ reflection symmetry analytic 
TABLE 1: Numbers of real and complex zeros of $E_{n}(x)$.

\begin{tabular}{|c|c|c|}
\hline Degree $n$ & Real zeros & Complex zeros \\
\hline 41 & 13 & 28 \\
\hline 42 & 10 & 32 \\
\hline 43 & 11 & 32 \\
\hline 44 & 12 & 32 \\
\hline 45 & 13 & 32 \\
\hline 46 & 14 & 32 \\
\hline 47 & 15 & 32 \\
\hline 48 & 12 & 36 \\
\hline 49 & 13 & 36 \\
\hline 50 & 14 & 36 \\
\hline 51 & 15 & 36 \\
\hline 52 & 16 & 36 \\
\hline 53 & 13 & 40 \\
\hline 54 & 14 & 40 \\
\hline 55 & 15 & 40 \\
\hline 56 & 16 & 40 \\
\hline 57 & 17 & 40 \\
\hline 58 & 14 & 44 \\
\hline 59 & 15 & 44 \\
\hline 60 & 16 & 44 \\
\hline 61 & 17 & 44 \\
\hline 62 & 18 & 44 \\
\hline 63 & 15 & 48 \\
\hline 64 & 16 & 48 \\
\hline 65 & 17 & 48 \\
\hline 66 & 18 & 48 \\
\hline 67 & 19 & 48 \\
\hline 68 & 20 & 48 \\
\hline 69 & 17 & 52 \\
\hline 70 & 18 & 52 \\
\hline 71 & 19 & 52 \\
\hline 72 & 20 & 52 \\
\hline
\end{tabular}

complex functions (Figures 1 and 2). The obvious corollary is that the zeros of $E_{n}(x)$ will also inherit these symmetries:

$$
\begin{aligned}
\text { If } E_{n}\left(x_{0}\right) & =0, \\
\text { then } E_{n}\left(1-x_{0}\right) & =E_{n}\left(x_{0}^{*}\right)=E_{n}\left(1-x_{0}^{*}\right)=0,
\end{aligned}
$$

where $*$ denotes complex conjugation (see Figures 1 and 2 ). By Theorem 4 and Conjecture 5 , the center of the structure of zeros is $1 / 2$.

The data concerning the numerical verification of Conjectures 6 and 7 are contained in Tables 1 and 2. See Table 1 for tabulated values of $R_{E_{n}(x)}$ and $C_{E_{n}(x)}$. First, we investigate the beautiful zeros of the $E_{n}(x)$ by using a computer. We plot the zeros of $E_{n}(x)$ for $n=70$ and $x \in \mathbb{C}$ (Figure 1). In Figure 1(a), the zeros of $E_{70}(x)$ structure are presented. In Figure 1(b), the real zeros of $E_{n}(x)$ structure are presented for $1 \leq n \leq 70$.

Stacks of zeros of $E_{n}(x)$ for $1 \leq n \leq 70$, forming a $3 \mathrm{D}$ structure, are presented (Figure 2). In Figure 2(a), we plot stacks of zeros of $E_{n}(x)$ for $1 \leq n \leq 70$. In Figure 2(b),
TABLE 2: Approximate solutions of $E_{n}(x)=0, x \in \mathbb{R}$.

\begin{tabular}{lc}
\hline Degree $n$ & $x$ \\
\hline 1 & 0.5 \\
2 & 0,1 \\
3 & $-0.366025404,0.5,1.36602540$ \\
4 & $-0.61803399,0,1,1.61803399$ \\
5 & $-0.6180,-0.6180,0.5,1.618,1.618$ \\
6 & 0,1 \\
7 & $-0.4977314,0.5,1.49773$ \\
8 & $-0.93233,0,1,1.9323$ \\
9 & $-1.21973,-0.50008,0.5,1.50008,2.21973$ \\
10 & $-0.46521,-1.01498,0,1,2.01498,2.36521$ \\
11 &
\end{tabular}

we draw $x$ and $y$ axes but no $z$ axis in three dimensions. In Figure 2(c), we draw $y$ and $z$ axes but no $x$ axis in three dimensions. In Figure 2(d), we draw $x$ and $z$ axes but no $y$ axis in three dimensions.

Our numerical results for the numbers of real and complex zeros of $E_{n}(x)$ are displayed (Table 1).

We observe a remarkably regular structure of the complex roots of Euler polynomials.

Next, we calculated an approximate solution satisfying $E_{n}(x), x \in \mathbb{R}$. The results are given in Table 2 .

From (2), we have

$$
\begin{aligned}
\sum_{n=0}^{\infty} & \left(E_{n}(x+1)+E_{n}(x)\right) \frac{t^{n}}{n !} \\
& =-2 \sum_{n=0}^{\infty}(-1)^{n+1} e^{(n+x+1) t}+2 \sum_{n=0}^{\infty}(-1)^{n} e^{(n+x) t}=2 e^{x t} \\
& =\sum_{n=0}^{\infty} 2 x^{n} \frac{t^{n}}{n !} .
\end{aligned}
$$

Comparing the coefficient of $t^{n} / n$ ! on both sides of (12), we get the following theorem.

Theorem 8. For any positive integer n, one has

$$
E_{n}(x+1)+E_{n}(x)=2 x^{n} .
$$

By (13), we have the following corollary.

Corollary 9. For $n \in \mathbb{N}$, one has

$$
E_{n}=-E_{n}(1) .
$$

\section{Julia Set of the Euler Polynomials}

In this section, we will present a series of diagrams showing the Julia set of the $E_{n}(x)$ and its related Mandelbrot set. Computations of the Julia and Mandelbrot sets of the $E_{n}(x)$ and observations of their properties are made. Let $\mathbb{C}$ denote the set of complex numbers and $\mathbb{C}_{\infty}=\mathbb{C} \cup\{\infty\}$. We generate graphic images using the software Mathematica. We define and construct orbits of points under the action of a complex 


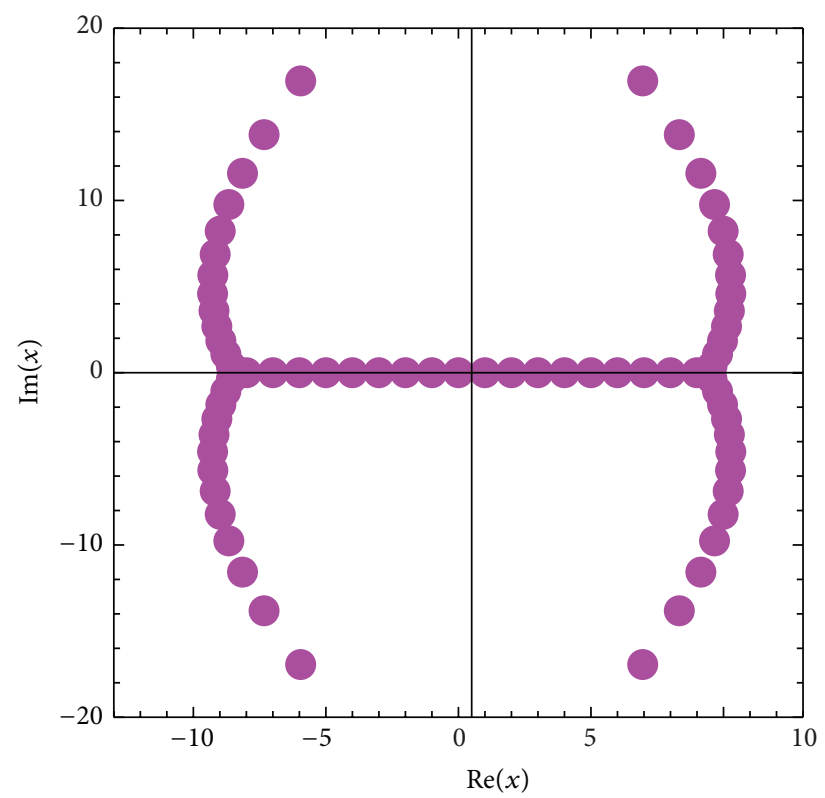

(a)

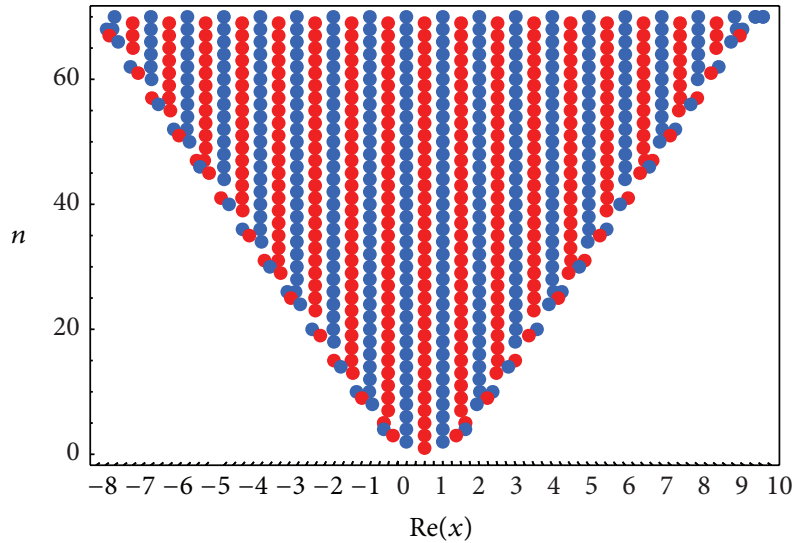

(b)

Figure 1: Zeros of $E_{n}(x)$.

function. Let $f: D \rightarrow D$ be a complex function, with $D$ being a subset of $\mathbb{C}$. The iterates of $f$ are the functions $f, f \circ f, f \circ f \circ f, \ldots$, which are denoted by $f^{1}, f^{2}, f^{3}, \ldots$ If $x \in \mathbb{C}$, then the orbit of $x$ under $f$ is the sequence $(x, f(x), f(f(x)), \ldots)$.

Definition 10 (see [21]). The orbit of the point $x$ under the action of the function $f$ is said to be bounded if there exists $M \in \mathbb{R}$ such that $\left|f^{n}(x)\right| \leq M$ for all $n \in \mathbb{N}$. If the orbit is not bounded, it is said to be unbounded.

Plotting the orbit of length 3 of the point $1+0.5 i$ under the action of the function $E_{3}(x)$, we get the results shown in Figure 3.

When plotting the orbit of a point, it is a good idea to start by plotting a few points, in order to obtain an idea if the orbit is bounded or unbounded. We see that the orbit of $E_{3}(x)$ is unbounded.

Definition 11 (see [21]). Let $f: D \rightarrow D$ be a complex function, with $D$ being a subset of $\mathbb{C}$. The point $z$ is said to be a fixed point of $f$ if $f(z)=z$. One also says that $\infty$ is a fixed point of $f$ if $|f(z)| \rightarrow \infty$ as $|z| \rightarrow \infty$.

Definition 12 (see [21]). A fixed point $p$ of $f(x)$ is an attractor if $\left|f^{\prime}(p)\right|<1$, a repeller if $\left|f^{\prime}(p)\right|>1$, and a neutral fixed point if $\left|f^{\prime}(p)\right|=1$.

As stated above, it can be proved that if $\alpha$ is an attracting fixed point of $f$, then there exists a neighbourhood $D$ of $\alpha$ such that if $b \in D$ the orbit of $b$ converges to $\alpha$. If $\alpha$ is a repelling periodic point of $f$, then there is a neighbourhood $N$ of $\alpha$ such that if $b \in N$ there are points in the orbit of
TABLE 3: Numbers of attractor, repeller, and neutral fixed points of $E_{n}(x)$.

\begin{tabular}{lccc}
\hline Degree $n$ & Attractor & Repeller & Neutral \\
\hline 2 & 0 & 1 & 1 \\
3 & 1 & 2 & 0 \\
4 & 0 & 1 & 3 \\
5 & 0 & 5 & 0 \\
6 & 0 & 6 & 0 \\
7 & 0 & 7 & 0 \\
8 & 0 & 8 & 0 \\
9 & 0 & 9 & 0 \\
10 & 0 & 10 & 0 \\
\hline
\end{tabular}

$b$ which are not in $N$. In the case of polynomials of degree greater than 0 and some rational functions, $\infty$ is also called an attracting fixed point, as, for each such function, $f$, there exists $R>0$ such that if $|x|>R$ then $f^{n}(x) \rightarrow \infty$ as $n \rightarrow \infty$.

Theorem 13 (fixed points of $E_{n}(x)$ ). The Euler polynomials $E_{3}(x)+0.2+0.5 i$ have one attractor fixed point (see Figure 4) at

$$
\alpha=0.347610414+0.283937229 i .
$$

Our numerical results for fixed point of $E_{n}(x)$ are displayed (Table 3). The results are obtained by Mathematica software.

Conjecture 14. For $n>3$, the Euler polynomials $E_{n}(x)$ have no attractor fixed point except for infinity. 


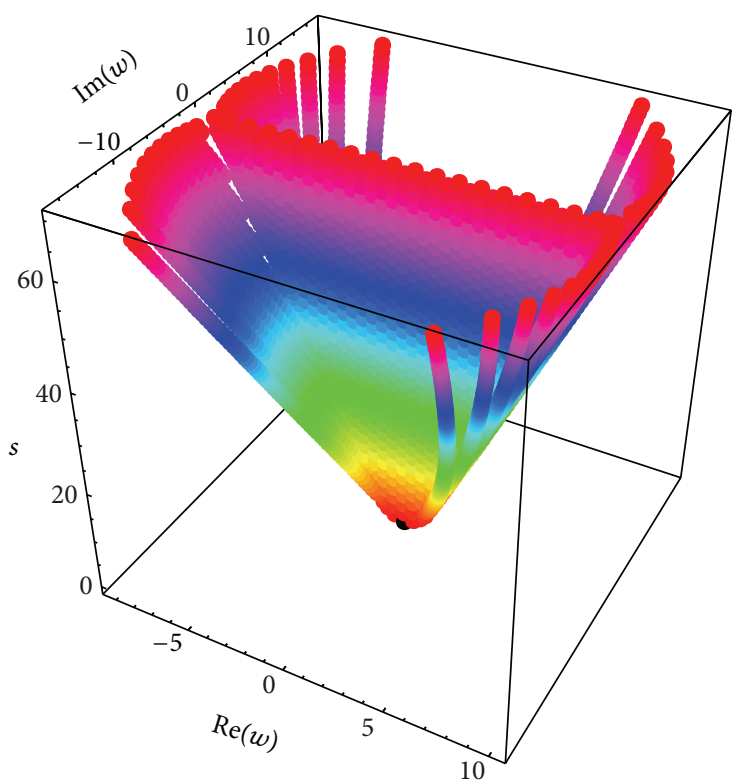

(a)

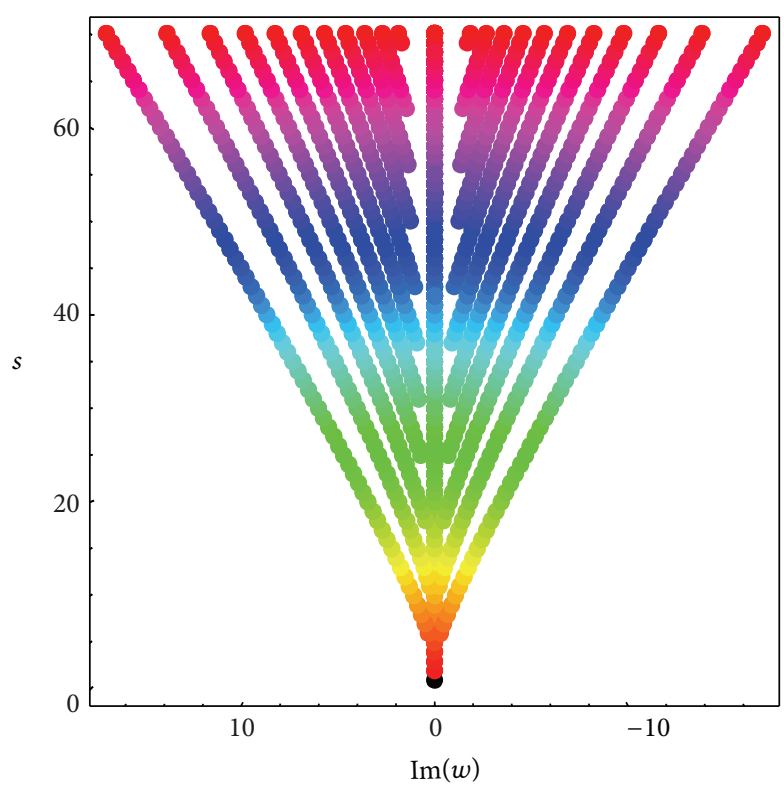

(c)

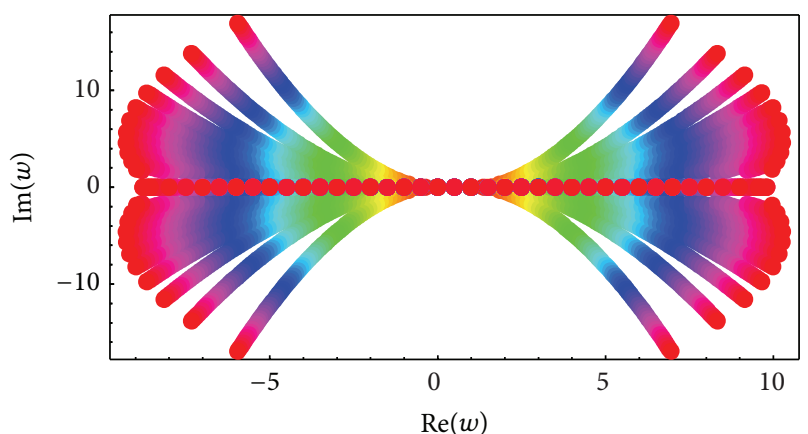

(b)

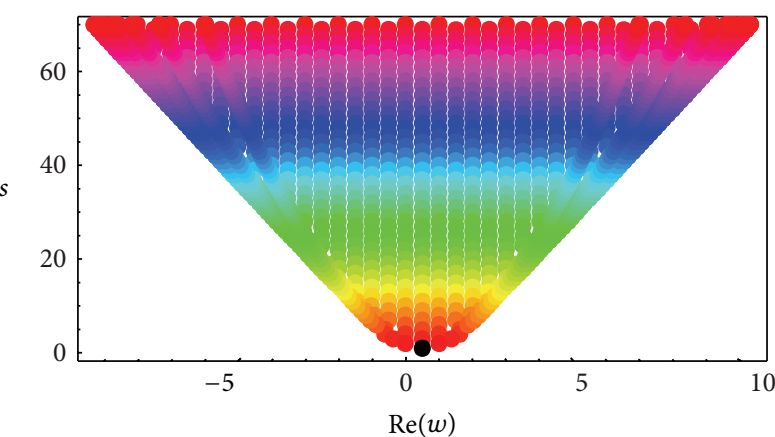

(d)

FIGURE 2: Stacks of zeros of $E_{n}(x)$ for $1 \leq n \leq 70$.

Definition 15 (basins of attraction). Let $f$ be a complex function with attracting fixed point $\alpha$. The basin of attraction of $\alpha$ under $f$ is defined to be the set

$$
\left\{z \in \mathbb{C} \mid f^{n}(z) \longrightarrow \alpha \text { as } n \longrightarrow \infty\right\} .
$$

Definition 16 (basins of attraction of infinity). If infinity is an attracting fixed point of $f$, then the basin of attraction of infinity is defined to be the set

$$
\left\{x \in \mathbb{C} \mid f^{n}(x) \longrightarrow \alpha \text { as } n \longrightarrow \infty\right\} .
$$

Let $f: \mathbb{C} \rightarrow \mathbb{C}$ be a polynomial in $x$ of degree $>2$ where $x \in \mathbb{C}$.
Definition 17 (Julia set). The filled Julia set of $f$ is the set

$$
K_{f}=\{x \in \mathbb{C} \mid \text { the orbit of } x \text { under } f \text { is bounded }\} .
$$

The Julia set of $f, J_{f}$, is the boundary of the filled Julia set of $f$.

Consider the family of functions $\left\{E_{3}(c): x \rightarrow E_{3}(x)+c \mid\right.$ $c \in \mathbb{C}\}$, where $c$ is a parameter. Julia sets can be divided into 2 classes. They are either connected or totally disconnected. Roughly speaking, a set which is connected is all in one piece (no breaks) while a set which is totally disconnected is like a cloud of dust particles, with its only connected components 


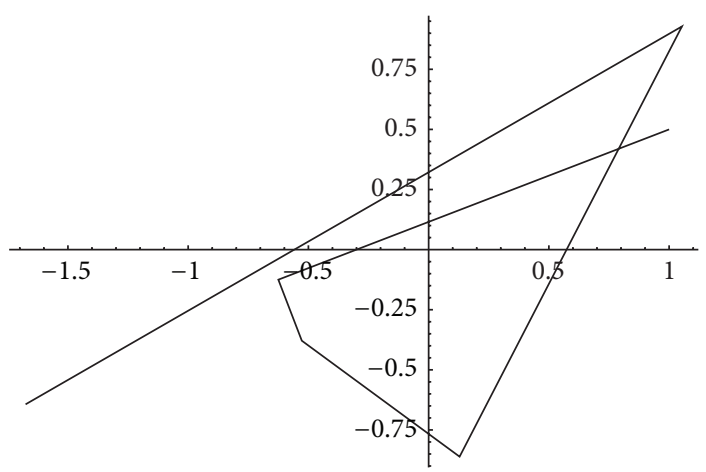

FIGURE 3: Unbounded orbit of $E_{3}(x)$.

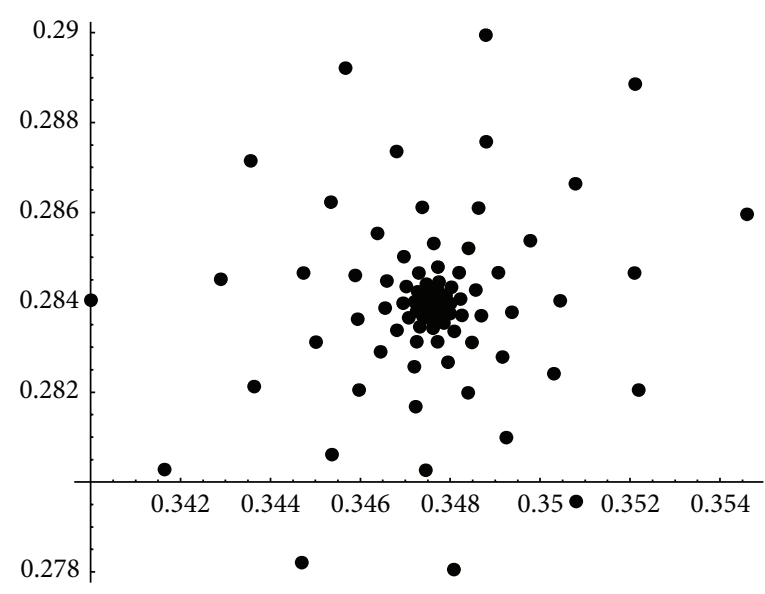

Figure 4: Attractor fixed point of $E_{3}(x)+0.2+0.5 i$.

being points. If $|c|<2$ then both types of Julia sets occur and both types are generally aesthetically pleasing. If $|c|>2$ then the Julia set of $E_{3}(c)$ is totally disconnected. This fact, together with the largeness of $c$, produces Julia sets which are rather dispersed and spread out and so not very pleasing. We show (a) in Figure 5(a) the Julia set of $E_{3}(-0.7280+0.0900 i)$ which is connected; (b) in Figure 5(b) the Julia set of $E_{3}(0.0340+$ $0.6250 i$ ) which is totally disconnected; (c) in Figure 5(c) the Julia set of $E_{3}(0.4010+0.0900 i)$ which is totally disconnected.

Examples of Julia sets for the family of $\left\{E_{5}(c): x \rightarrow\right.$ $\left.E_{5}(x)+c \mid c \in \mathbb{C}\right\}$ are shown in Figure 6.

Newton tells us to consider the dynamical system

$$
\left\{\mathbb{C}_{\infty}: R(x)=x-\frac{S(x)}{S^{\prime}(x)}\right\} .
$$

$R$ is called the Newton iteration function of $S$. It can be shown that the fixed points of $R$ are zeros of $S$ and that all fixed points of $R$ are attracting. $R$ may also have one or more attracting cycles. To obtain the Julia set of $R$, plot, in white, the set $J$ of points whose orbits do not converge, and plot the set $U$ of remaining points whose orbits converge in a different coloring. The boundary of the set $J$ or of the set $U$ is the Julia set of $R$.

Consider the Euler polynomial $E_{4}(x)$ for $x \in \mathbb{C}$. There are four distinct complex numbers, $a_{i}(i=1,2,3,4)$, such that
$E_{4}\left(a_{i}\right)=0$. Newton's method provides a means to compute them. We obtain $a_{1}=0, a_{2}=2, a_{3}=(1 / 2)(1-\sqrt{5})$, and $a_{4}=(1 / 2)(1+\sqrt{5})$. Then Newton tells us to consider the dynamical system

$$
\left\{\mathbb{C}_{\infty}: R(x)=x-\frac{E_{4}(x)}{E_{4}^{\prime}(x)}\right\} .
$$

The general expectation is that a typical orbit $\left\{R^{n}(x)\right\}$, which starts from an initial "guess" $x_{0} \in \mathbb{C}$, will converge to one of the roots of $E_{4}(x)$. If we choose $x_{0}$ close enough to $a_{i}$ then it is readily proved that

$$
\lim _{n \rightarrow \infty} R^{n}\left(x_{0}\right)=a_{i}, \quad \text { for } i=1,2,3,4 .
$$

Given a point $x_{0}$ in the plane, we wish to find out if the orbit of $x_{0}$ under the action of $R(x)$ does or does not converge to one of the roots of the equation, and if so, which one.

When $R(x)$ is applied to $x_{0}$, the orbit of $x_{0}$ under the action of $R$ is calculated until the absolute value of the last 2 iterations differs by an amount less than $10^{-10}$ or until 30 iterations have been carried out. The output is the last orbit value calculated. We construct a function which assigns one of 4 colors to each point in the plane, according to the outcome of $R$. We assign the red, yellow, green, and blue to $x_{0}$ if its orbit converges to $0,1,(1 / 2)(1-\sqrt{5}),(1 / 2)(1+$ 


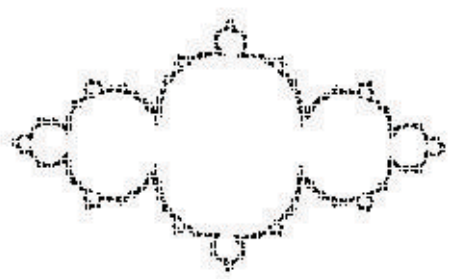

(a)

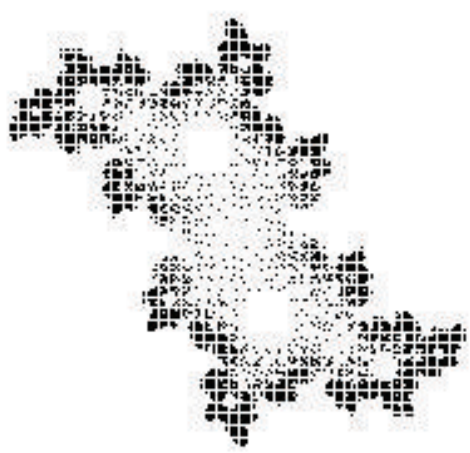

(b)
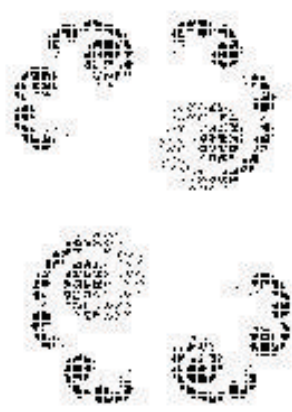

(c)

FIGURE 5: Julia sets $E_{3}(c)$.
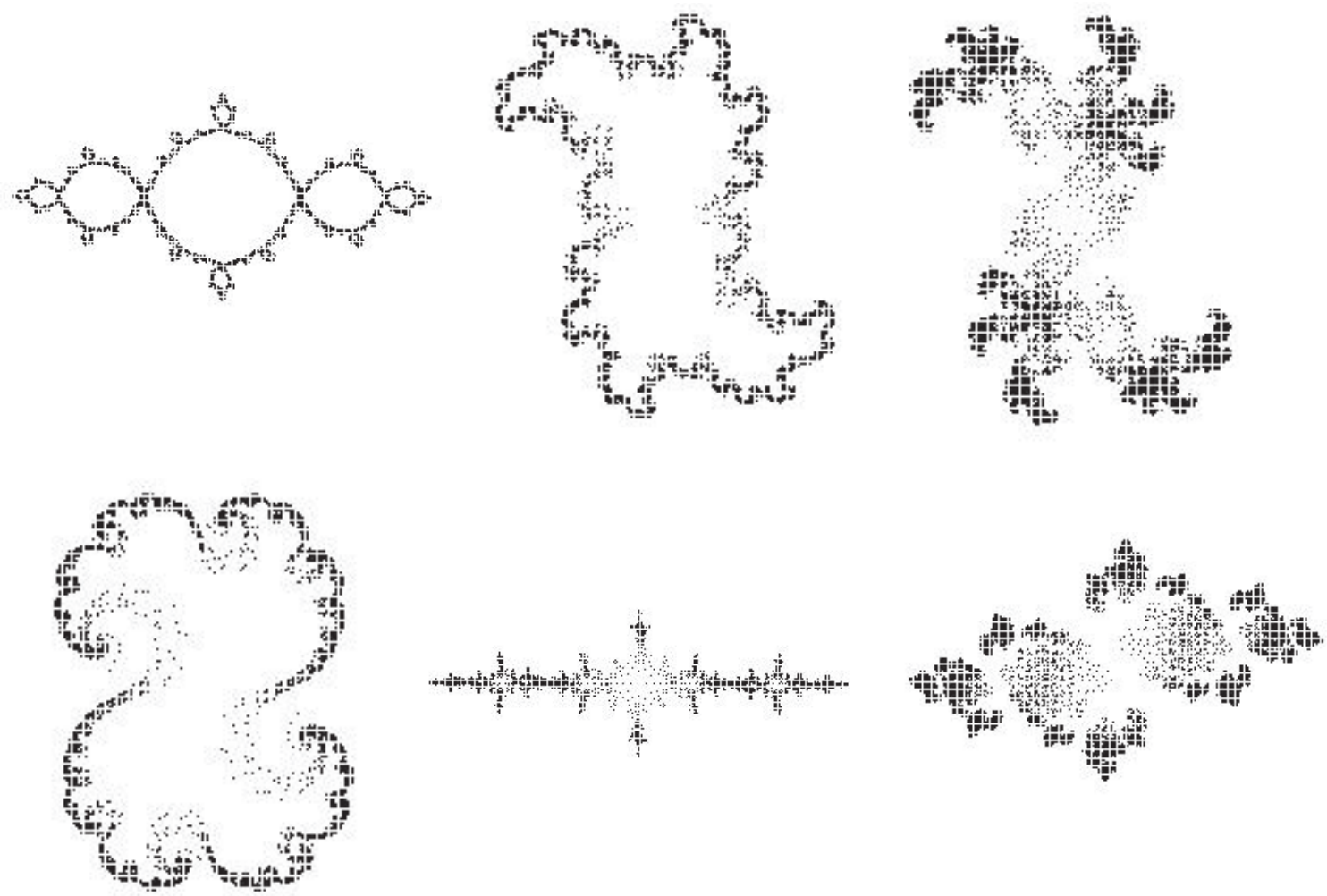

Figure 6: Julia sets $E_{5}(c)$

$\sqrt{5})$, respectively. We assign the black to it if the orbit does not converge to any of the points (Figure 7 ). The viewing windows are $\{(x, y):-6 \leq x \leq 6,-6 \leq y \leq 6\}$. In Figure 7 , the red region represents part of the basin of attraction of $a_{1}=0$.

As the Newton iteration function of $E_{4}(x)$ is of degree 4 , we plot, in white, the set of points whose orbits do not converge. The Julia set will be the boundary of the set of black points (see Figure 8). The viewing windows are $\{(x, y)$ : $-5.5 \leq x \leq 5.5,-5.5 \leq y \leq 5.5\}$.

A zoom-in on a version of Figure 8, in which the contours and contour lines are colored, is shown in Figure 9. The viewing windows are $\{(x, y):-2.5 \leq x \leq 3.5,-2.5 \leq y \leq$ $2.5\}$.

This color palette explains the coloring of Figure 9. For example, points which escape after 1 to 30 iterations are colored red to green. Points which do not escape after 30 iterations and so are taken to be in the filled Julia set are colored black (Figure 10).

The abovementioned rapid change can also be illustrated by applying the three-dimensional structure to the escapetime function (Figure 11). The viewing windows are $\{(x, y)$ : $-5.5 \leq x \leq 5.5,-5.5 \leq y \leq 5.5\}$. When $R(x)$ is applied to $x_{0}$, the orbit of $x_{0}$ under the action of $R$ is calculated until the 


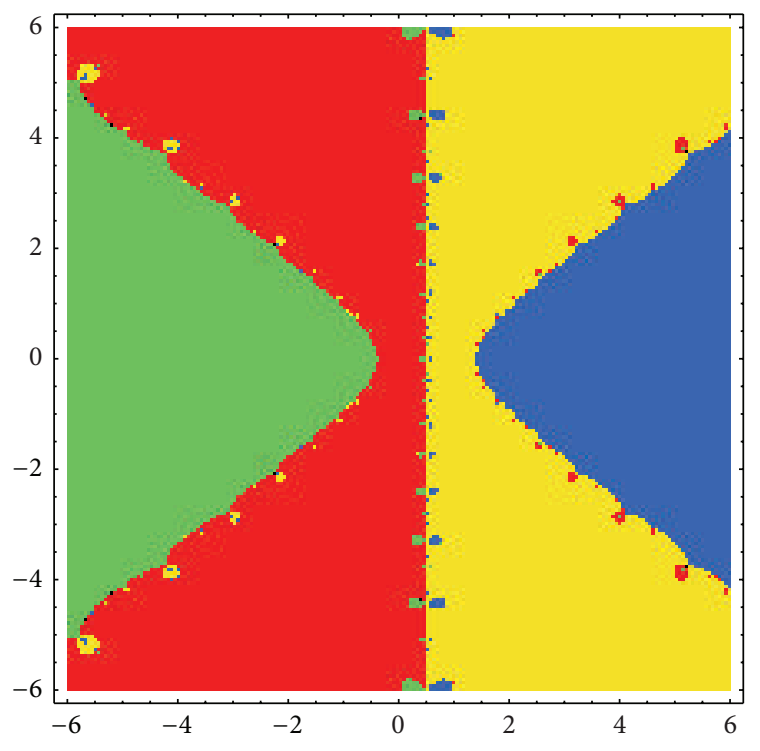

FIgURE 7: Orbit of $x_{0}$ under the action of $R$ for $E_{4}(x)$.

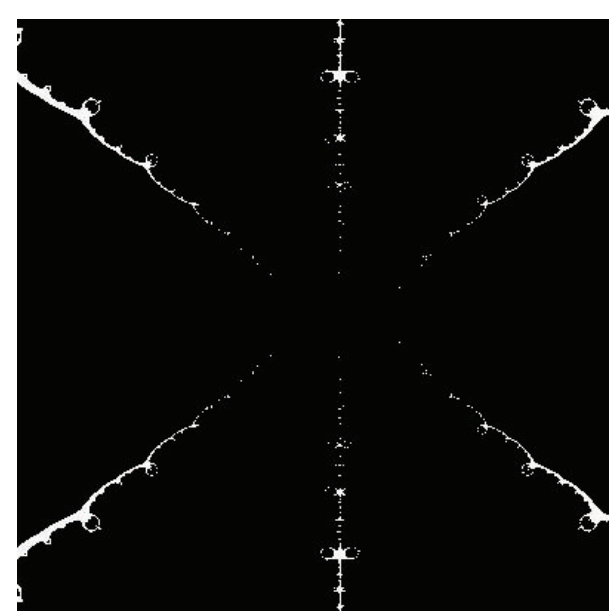

Figure 8: Julia set of $R(x)$ for $E_{4}(x)$.

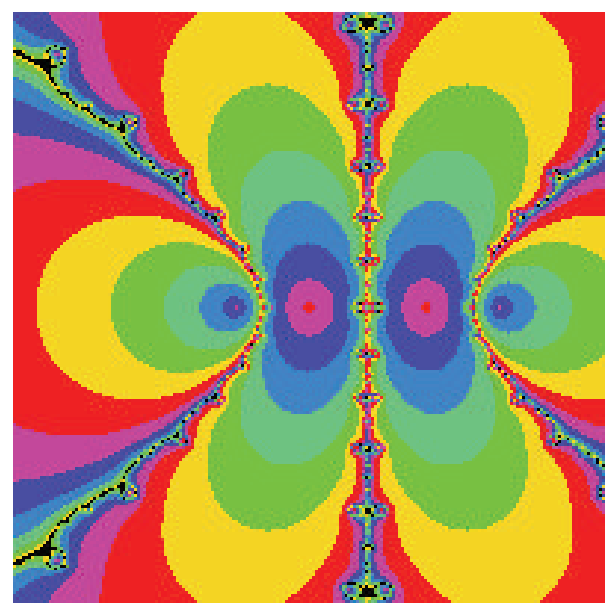

Figure 9: Julia set of $R(x)$ for $E_{4}(x)$.

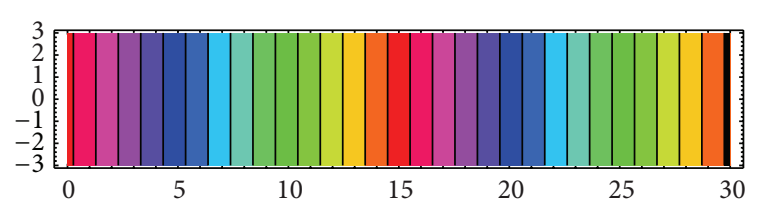

FIgURE 10: Palette for escaping points.

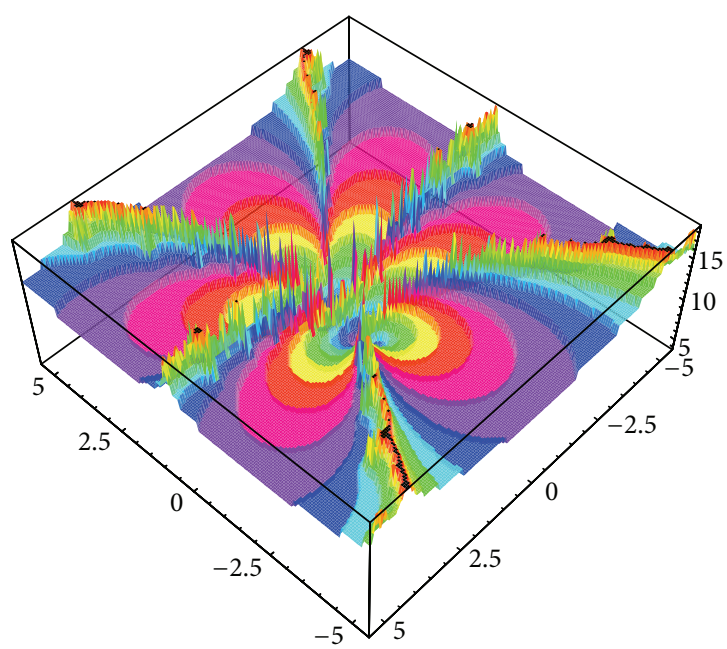

FIGURE 11: 3D structure of Julia set of $R(x)$ for $E_{4}(x)$.

absolute value of the last 2 iterations differs by an amount less than $10^{-10}$ or until 30 iterations have been carried out. The output is the last orbit value calculated.

\section{Conflict of Interests}

The authors declare that there is no conflict of interests regarding the publication of this paper.

\section{Acknowledgment}

This work was supported by the NRF (National Research Foundation of Korea) Grant funded by the Korean Government (NRF-2013-Fostering Core Leaders of the Future Basic Science Program).

\section{References}

[1] M. Alkan and Y. Simsek, "Generating function for q-Eulerian polynomials and their decomposition and applications," Fixed Point Theory and Applications, vol. 2013, article 72, 14 pages, 2013.

[2] R. Ayoub, "Euler and the zeta function," The American Mathematical Monthly, vol. 81, pp. 1067-1086, 1974.

[3] A. Bayad, "Modular properties of elliptic Bernoulli and Euler functions," Advanced Studies in Contemporary Mathematics, vol. 20, pp. 389-401, 2010.

[4] J. Y. Kang, H. Y. Lee, and N. S. Jung, "Some relations of the twisted $q$-Genocchi numbers and polynomials with weight $\alpha$ and weak weight $\beta$," Abstract and Applied Analysis, vol. 2012, Article ID 860921, 9 pages, 2012. 
[5] M.-S. Kim and S. Hu, "On p-adic Hurwitz-type Euler zeta functions," Journal of Number Theory, vol. 132, no. 12, pp. 29773015, 2012.

[6] T. Kim, C. S. Ryoo, L. C. Jang, and S. H. Rim, "Exploring the qriemann zeta function and $q$-bernoulli polynomials," Discrete Dynamics in Nature and Society, no. 2, pp. 171-181, 2005.

[7] T. Kim and S.-H. Rim, "Generalized Carlitz's euler numbers in the p-adic number field," Advanced Studies in Contemporary Mathematics, vol. 2, pp. 9-19, 2000.

[8] T. Kim, "Euler numbers and polynomials associated with zeta functions," Abstract and Applied Analysis, vol. 2008, Article ID 581582, 11 pages, 2008.

[9] H. Ozden and Y. Simsek, "A new extension of q-euler numbers and polynomials related to their interpolation functions," Applied Mathematics Letters, vol. 21, no. 9, pp. 934-939, 2008.

[10] H. Ozden and Y. Simsek, "Interpolation function of the $(h, q)$ extension of twisted Euler numbers," Computers \& Mathematics with Applications, vol. 56, no. 4, pp. 898-908, 2008.

[11] K. H. Park, S.-H. Rim, and E. J. Moon, "On Genocchi numbers and polynomials," Abstract and Applied Analysis, vol. 2008, Article ID 898471, 7 pages, 2008.

[12] C. S. Ryoo, T. Kim, and R. P. Agarwal, "A numerical investigation of the roots of $q$-polynomials," International Journal of Computer Mathematics, vol. 83, no. 2, pp. 223-234, 2006.

[13] C. S. Ryoo and Y. S. Yoo, "A note on Euler numbers and polynomials," Journal of Concrete and Applicable Mathematics, vol. 7, no. 4, pp. 341-348, 2009.

[14] C. S. Ryoo, "A numerical computation of the roots of $q$ euler polynomials," Journal of Computational Analysis and Applications, vol. 12, no. 1, pp. 148-156, 2010.

[15] C. S. Ryoo, "Analytic continuation of EULer polynomials and the EULer zeta function," Discrete Dynamics in Nature and Society, vol. 2014, Article ID 568129, 6 pages, 2014.

[16] C. S. Ryoo, "Zeros of analytic continued $q$-euler polynomials and q-euler zeta function," Journal of Applied Mathematics, vol. 2014, Article ID 806239, 7 pages, 2014.

[17] C. S. Ryoo, H. I. Kwon, J. Yoon, and Y. S. Jang, "Fourier series of the periodic Bernoulli and Euler functions," Abstract and Applied Analysis, vol. 2014, Article ID 856491, 4 pages, 2014.

[18] Y. Simsek, "Generating functions of the twisted Bernoulli numbers and polynomials," Advanced Studies in Contemporary Mathematics, vol. 16, pp. 251-257, 2008.

[19] Y. Simsek, "Twisted $(h, q)$-Bernoulli numbers and polynomials related to twisted $(h, q)$-zeta function and L-function," Journal of Mathematical Analysis and Applications, vol. 324, no. 2, pp. 790-804, 2006.

[20] Y. Simsek, "Complete sum of products of $(h, q)$-extension of Euler polynomials and numbers," Journal of Difference Equations and Applications, vol. 16, no. 11, pp. 1331-1348, 2010.

[21] C. Getz and J. M. Helmstedt, Graphics with Mathematica: Fractals, Julia Sets, Patterns and Natural Forms, Elsevier, Amsterdam, The Netherlands, 2004. 


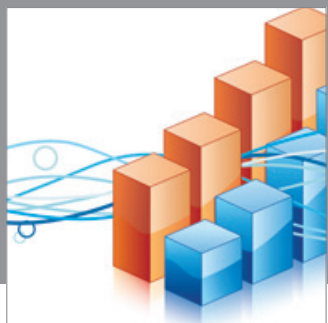

Advances in

Operations Research

mansans

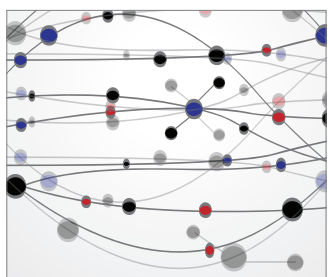

The Scientific World Journal
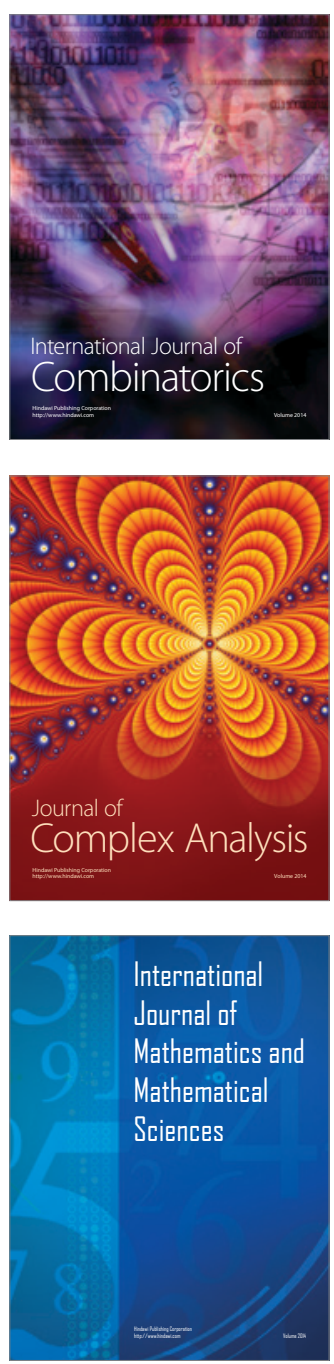
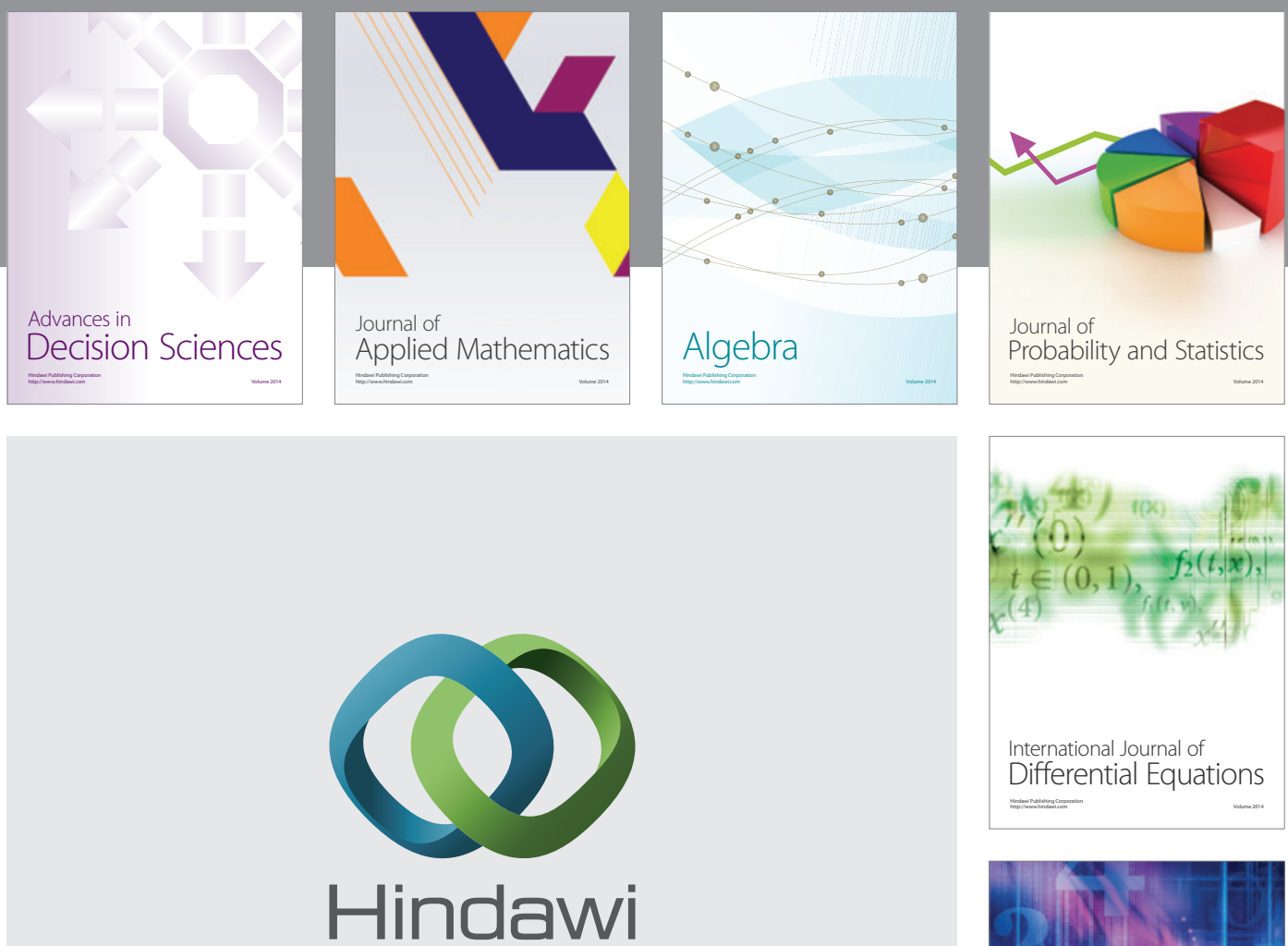

Submit your manuscripts at http://www.hindawi.com
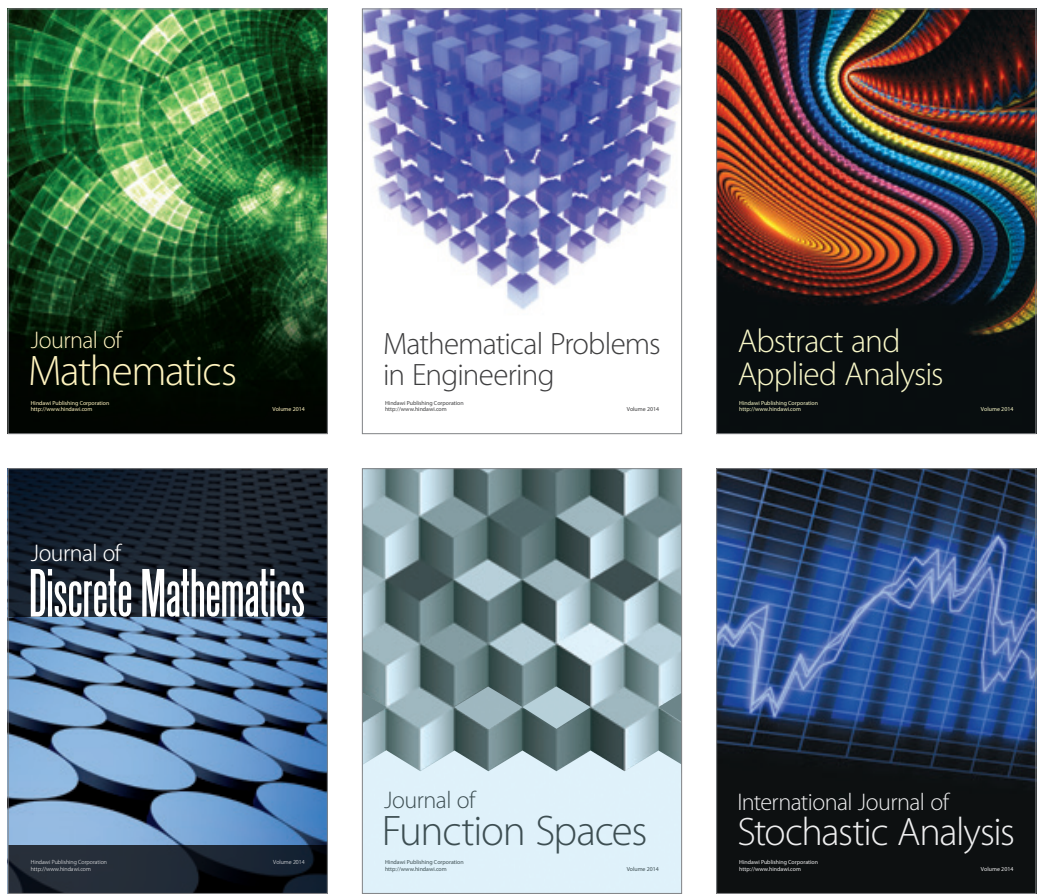

Journal of

Function Spaces

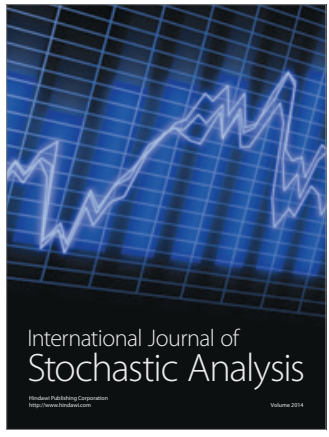

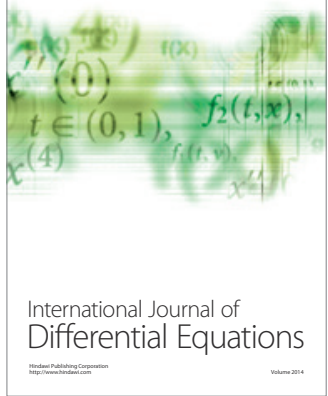
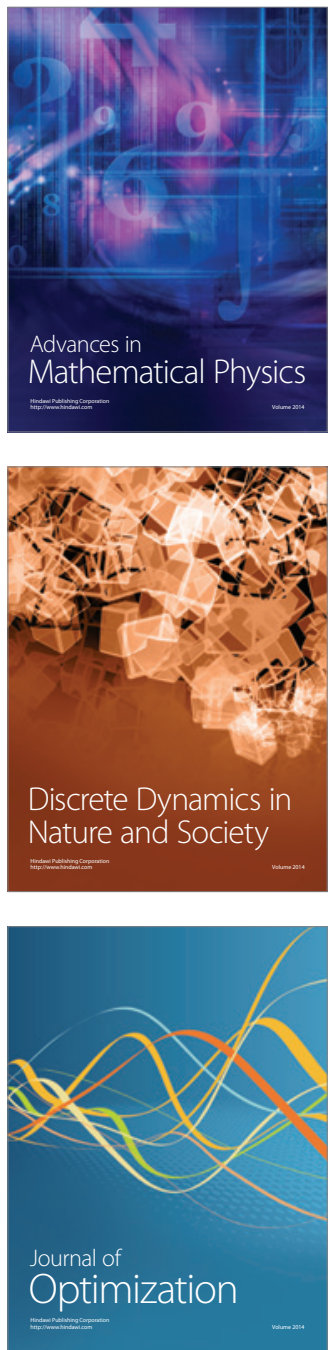